phenomenal success in making a difference in practice. Millions of animals are now moving through slaughterhouses more calmly because of what she has seen through her autistic eyes.

In some ways, this book is profoundly shocking, or at least it would be if it were not written by an autist. "Autistic people are closer to animals than normal people are," she writes. "Autistic peoples' frontal lobes almost never work as well as normal people's do, so our brain function ends up being somewhere in between human and animal." Imagine anyone else saying that and getting away with it. But part of the power of this book is its innocence (her word too) and its genuine insights into a completely different way of thinking and seeing. Political correctness is not part of her world.

Grandin also states categorically that she doesn't have an unconscious and cannot repress unwelcome thoughts or emotions. This is one of many areas in which she has difficulty understanding normal people. She cannot understand why they deny what seems perfectly obvious to her, such as that something isn't going to work or shouldn't be said because it would offend people. Hearing her side of the story is a bit like being introduced to another culture by someone from that culture who has taken the trouble to find out which bits you are going to struggle to understand. Grandin straddles two worlds and, remarkably, can operate in both. If we want to understand her world, some of our preconceptions about what can and cannot be said will also have to be toppled. Being shocked out of our usual way of thinking is part of the process of understanding what it is like to be her.

At times, it is difficult to work out whether this is a book about animal behaviour with insights from autism, or a book about autism that uses animal behaviour to explain what it is like to be autistic. A major achievement of the book is that is both, and it should be read as such. If you disagree with some of the things she says about animal behaviour or brain function, you keep going because of the fascinating insights you are gaining into her experiences as an autist; and if you find yourself disagreeing with what she says about autism, you have the testimony of a unique human being about her life with animals.

Catherine Johnson has put Grandin's words into a form that is accessible to readers without any prior knowledge of either autism or animal behaviour. At the same time, people working in these areas will be given insights into the connections between these apparently diverse fields that will stick in the mind and change the way we look at both.

Marian Stamp Dawkins is in the Department of Zoology, University of Oxford, South Parks Road, Oxford OX1 3PS, UK.

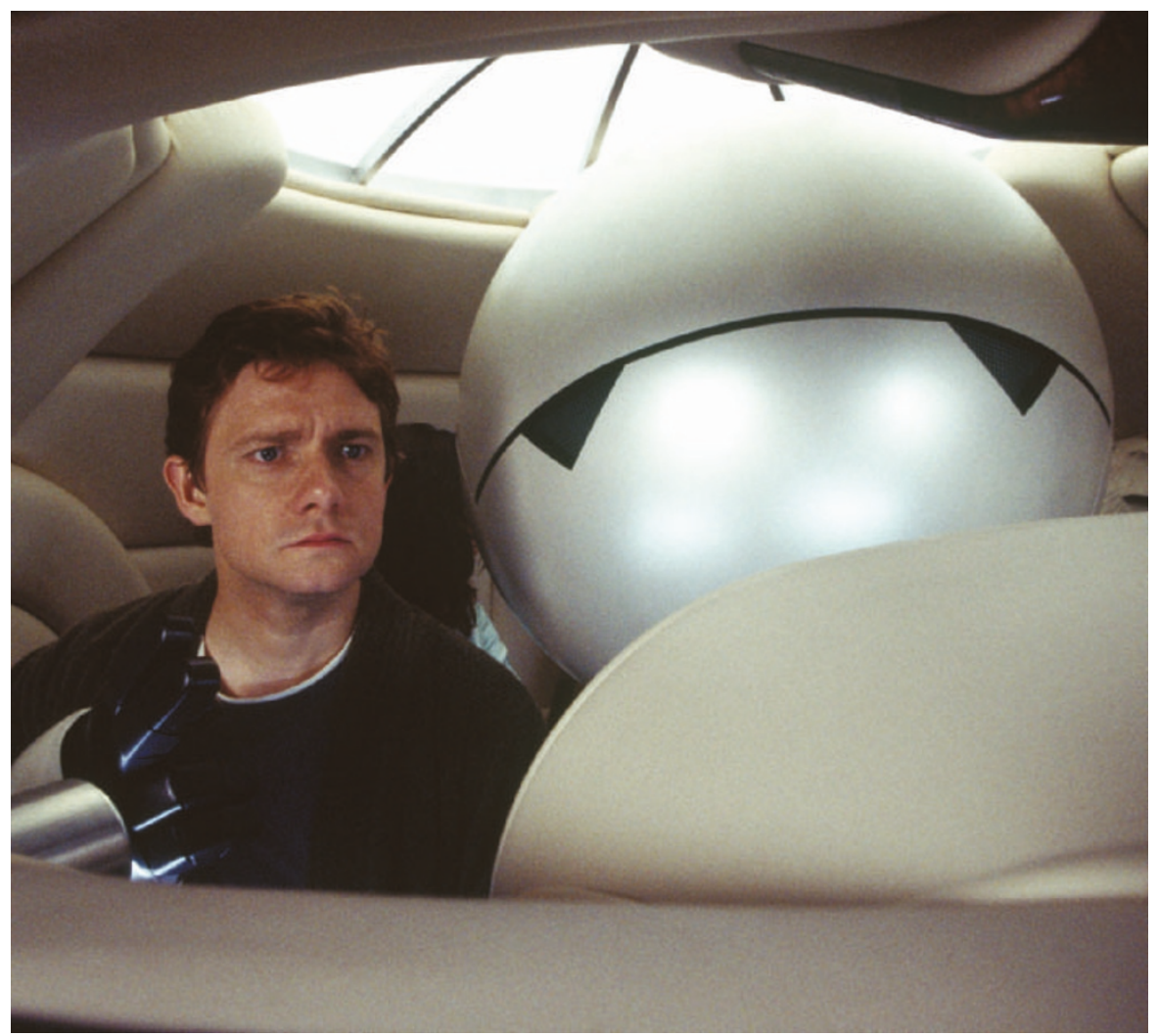

Guided tour: Arthur Dent and Marvin the Paranoid Android hitchhike through the galaxy.

\section{Don't panic!}

\section{The Science of the Hitchhiker's Guide to the Galaxy \\ by Michael Hanlon \\ Macmillan Science: 2005. 256 pp. \\ $£ 16.99, \$ 24.95$}

\section{Joanne Baker}

Your brief: to explain the science of Life, the Universe and Everything. It's quite a challenge. But Michael Hanlon pulls it off with wit, energy and style.

Timed to coincide with the release of the film adaptation of Douglas Adams' famous book series The Hitchhiker's Guide to the Galaxy, Hanlon's guide to the Guide takes the general reader on a grand tour of the outer reaches of modern scientific reality. Alien life, quantum physics and the history of the Universe are just as mind-boggling and weird now as they were to Adams in the 1970s, when he imagined Arthur Dent's escapades. Hanlon even manages to explain the unexplainable, such as the eye-popping shock of the total-perspective vortex.

Hanlon obviously enjoyed writing this book. It's not often that science writers get to rant about the non-existence of God, to explain the sudden appearance of a whale from a quantum fluctuation, or to ponder the genetic modification of animals to produce guilt-free meat. Adopting Adams' witty, punchy style, Hanlon's guide is a fun and vivid read. The science twinkles a little more than usual in such a zany setting.

Although he tackles a wide range of cutting-edge topics with depth and authority, Hanlon has chosen the most obvious Hitchhiker destinations for his own scientific tour. The Restaurant at the End of the Universe prompts a discussion of the fate of the Universe; the babel fish yields a chapter on translation software; and time travel, parallel universes and black holes are well-trodden avenues. But when Hanlon does venture off-piste, he is a reassuring and insightful travelling companion, even if he often leaves the Guide behind. More references to it and amusing quotes could have added to the entertainment.

Readers familiar with the original Hitchhiker's Guide might have enjoyed more subtle tie-ins and a little more background about Adams himself, his peculiar ideas and influences. Hanlon briefly sets the context, but leaves such dialogue to others. The book also lacks comparisons with other contemporary science fiction. Aimed fair and square at the popular-science market, Hanlon's book may not satisfy die-hard science-fiction buffs. But the ghost of Adams is lurking in the pages.

See the film and buy the books. Don your striped jacket, bathrobe or spare head, and keep a towel handy. And above all — Don't Panic. With Hanlon's quirky book you are in safe hands.

Joanne Baker is in the Astrophysics Department, University of Oxford, Denys Wilkinson Building, Keble Road, Oxford OX10 3RH, UK. 